\title{
Repères d'une philosophie française de la subjectivité réflexive en littérature
}

\author{
Andrea Bellia \\ University of Melbourne
}

\section{Résumé}

Cet article présente seulement quelques idées d'une pensée philosophique qui a rayonné en France entre les deux guerres et qui a connu malheureusement un déclin considérable à la suite de l'influence de la philosophie allemande. La philosophie réflexive, connue ainsi par sa méthode de réflexion du sujet, vise à repérer le fondement métaphysique du moi audelà de tout déterminisme lié aux faits psychologiques objectivables. Le moi est saisi par un retour de la pensée sur elle-même comme causalité spirituelle de tout acte volitif. À partir du cogito cartésien, Maine de Biran a été l'inspirateur de cette méthode philosophique de grand intérêt qui fonde une métaphysique de la conscience en tant que dimension première d'une philosophie de la subjectivité. Une relecture de la célèbre œuvre de Marcel Proust, à la lumière de cette lignée de pensée, nous révèle l'influence de la philosophie réflexive en littérature. Ceci remettrait en avant une philosophie éclipsée mais de grande valeur pour le monde francophone contemporain et ainsi jetterait une nouvelle lumière sur la critique littéraire de notre époque.

Mots-clés : Philosophie réflexive, Moi, Conscience, Métaphysique, Littérature.

«La vraie vie, la vie enfin découverte et éclaircie, la seule vie par conséquent pleinement vécue, c'est la littérature ».

(Marcel Proust, 1989 : 474) 


\section{Introduction}

Une école de pensée florissante en France entre les deux guerres et tombée totalement dans l'oubli - bien qu'elle demeure dissimulée dans le développement des sciences humaines et dans la littérature - mérite aujourd'hui une remise en valeur à travers une reconstruction épistémologique ciblée, même si cela n'est pas une tâche simple à accomplir. Il s'agit d'une philosophie française de la subjectivité, connue comme philosophie réflexive ou philosophie à la seconde puissance, un itinéraire de pensée peu connu et encore moins étudié. Par son analyse du moi, source de tout acte volitif, elle se caractérise comme philosophie de la subjectivité pure et de l'intériorité réflexive.

J'essaierai de présenter quelques traits de cette philosophie réflexive, tout en sachant que les orientations différentes des auteurs qui la représentent ne permettent pas d'établir en quelques lignes et de manière systématique, une pensée assez difficile à rendre et à recevoir dans son ensemble.

Toute démarche ayant pour but l'identification de repères de cette philosophie serait d'importance capitale pour mieux comprendre d'autres expressions culturelles de la même époque. En effet, une analyse critique de la littérature d'une époque, isolée de toute source de pensée inspiratrice, se révèlerait une démarche naïve et décontextualisée. Nous verrons plus loin comment l'œuvre de l'un des plus grands auteurs de la littérature française, Marcel Proust, n’est pas étrangère à la philosophie de son époque, une philosophie rapidement disparue et difficile à identifier. 
Toute expression du phénomène francophone, que ce soit dans la pensée, dans l'art ou dans le langage, mérite d'être comprise dans son intégralité et sa complexité constitutives.

\section{La philosophie réflexive en France}

Le volume 1 de l'Essai sur la philosophie réflexive paru en 1971, puis le volume 2 paru en 1974 de Ludovic Robberechts ne comblent pas la lacune en ce qui concerne la particularité méthodologique et la visée investigatrice de cette philosophie française. L'œuvre de Robberechts nous présente des auteurs clés de la philosophie réflexive mais elle ne nous offre pas une exposition systématique de cette pensée. Je n'ai pas non plus la prétention d'offrir ici une reconstruction épistémologique de la philosophie réflexive française, sinon quelques éléments influents qu'on retrouve aussi en littérature.

L'univers académique, comme toute réalité mondaine, subit l'orientation de la mode, et depuis l'événement «Husserl», le langage qui domine dans le monde universitaire et éditorial est celui qui fait référence aux domaines de la phénoménologie et de l'herméneutique. Des auteurs comme Ravaisson, Lachelier, Lagneau et d'autres, sont presque inconnus et pour susciter l'intérêt pour une philosophie qui n'est plus à la mode, il faudrait peut-être considérer un penseur qui domine la scène académique actuelle. Le célèbre philosophe français Paul Ricœur ${ }^{3}$ pourrait sans doute nous rendre ce service et nous conduire à la découverte de ce courant de pensée.

\footnotetext{
${ }^{3}$ Mon entrée en matière par le biais de ce penseur français de réputation mondiale trouve aussi son fondement dans une expérience vécue en 1997, quand Paul Ricœur lui-même m'a fait l'honneur de me recevoir dans sa maison à Châtenay-Malabry et de discuter avec moi sur sa
} 
Ricœur a commencé son activité spéculative, séduit par cette philosophie réflexive et il portera longtemps les empreintes de cette pensée tout en faisant place à un domaine d'investigation philosophique assez vaste et complexe.

En 1934, Ricœur soutient son mémoire de maîtrise sous le titre: $L a$ méthode réflexive appliquée au problème de Dieu chez Lachelier et Lagneau. Bien que dans ce mémoire Ricœur ne reconstruise pas une histoire de la méthode réflexive, il révèle tout de même comment l'approche réflexive de Lachelier et de Lagneau conduit à une recherche de Dieu à partir du moi et donc à partir d'une philosophie de la subjectivité.

Plus tard, Ricœur s'investira dans la réalisation des préfaces des ouvrages d'un des derniers et plus grands représentants de la philosophie réflexive française : Jean Nabert. Nous avons donc sa préface de la deuxième édition de l'Essai sur le mal en 1959, la préface de la deuxième édition des Éléments pour une éthique en 1962 et la préface du Désir de Dieu en 1970. L'expérience intérieure de la liberté, thèse soutenue par Nabert en 1924, sera publiée en 1994 aux Presses Universitaires de France avec encore une préface rédigée par Ricœur sous le titre: L'arbre de la philosophie réflexive. Une analyse attentive de ces préfaces ainsi que de ses articles comme L'acte et le signe selon Jean Nabert ${ }^{4}$ et Négativité et affirmation originaire ${ }^{5}$, nous montre l'attachement de Ricœur non seulement à ce représentant de la philosophie réflexive française mais aussi à ce

vision de la philosophie réflexive en France et sur l'impact que cette pensée avait eu dans son itinéraire spéculatif.

${ }^{4} \mathrm{P}$. Ricœur, L'acte et le signe selon Jean Nabert.

${ }^{5}$ Ceci a été intégré dans la deuxième édition de P. Ricœur, Histoire et vérité (1955 : 336-360). 
courant philosophique éclipsé et cependant encore perceptible dans d'autres formes d'expression de la pensée.

La philosophie réflexive sera malheureusement étouffée par la phénoménologie husserlienne mais aussi par la philosophie herméneutique. Ricœur suit le même parcours : son itinéraire spéculatif initial se situe dans la ligne d'une philosophie réflexive, puis il se voit appartenant à l'école phénoménologico-herméneutique, une variante herméneutique de la phénoménologie husserlienne (Paul Ricœur, 1986: 25). En effet, bien que Ricœur ait reconnu Nabert comme son maître, sa pensée témoigne d'une continuité avec ce dernier, puis d'un dépassement et puis encore d'un total éloignement en ce qui concerne le domaine d'investigation et de réflexion.

Une recherche relative à la genèse de la philosophie réflexive à partir de Descartes et Maine de Biran pour arriver enfin aux auteurs clés d'une pensée qui donne le primat à la vie de la conscience comme Lachelier, Lagneau, Ravaisson, Lavelle, Lequier, Le Senne, Madinier, Forest et surtout Nabert, montrera la richesse et l'intérêt de cette exploration philosophique. Bien entendu, il n'est pas possible d'examiner ici la pensée de ces auteurs mais je tâcherai tout de même de repérer juste quelques idées de Lachelier et de Nabert.

La philosophie réflexive se voit enracinée dans le cogito cartésien qui marque dès lors le tournant décisif pour toute philosophie de la subjectivité. Le sujet lui-même, dans sa capacité de penser, devient à partir de l'époque contemporaine une source inépuisable de réflexions ainsi que d'orientations de pensées assez variées. 
Jean-Paul Sartre, dans une conférence donnée à Paris en 1945 sous le titre L'existentialisme est un humanisme ${ }^{6}$, affirme que le cogito est la vérité absolue de la conscience dans son orientation tournée sur elle-même « car, en dehors du cogito cartésien, tous les objets sont seulement probables, et une doctrine de probabilités, qui n'est pas suspendue à une vérité, s'effondre dans le néant ${ }^{7}$. Pour Sartre, la vérité absolue est un produit du cogito en tant que garant du moi et elle se saisit dans l'acte même d'une prise de conscience de soi, de manière directe et sans intermédiaires. Mais pour une entrée en matière plus spécifique d'une philosophie française de la subjectivité qui se caractérise comme philosophie réflexive, il faut sans doute étudier d'abord le travail de Jules Lachelier ${ }^{8}$ sous le titre «Psychologie et métaphysique » publié originairement en mai 1885 dans la Revue philosophique.

Il paraît que malgré une certaine rigueur de discrétion de la part de Lachelier contre toute volonté de rendre publiques ses écrits et ses notes de recherche, un fonds a été constitué à la Bibliothèque Mazarine dans le $6^{\mathrm{e}}$ arrondissement de Paris. Ceci permettrait que ce penseur soit davantage étudié dans le contexte de la philosophie réflexive française et également dans son rapport à d'autres philosophes qui partagent la même orientation.

Voici ce que Lachelier (1949 : 68) écrit dans Psychologie et métaphysique : «Il ne nous reste plus qu'à nous expliquer une dernière fois sur les deux questions, évidemment connexes, du moi et de la liberté ». Ceux-ci sont deux

\footnotetext{
${ }^{6}$ La conférence a été publiée en 1946 aux Éditions Nagel.

${ }^{7}$ Ma référence à la conférence de Sartre ne veut nullement soutenir le contenu général de son propos mais plutôt mettre l'accent sur le fait qu'une philosophie de la subjectivité domine l'espace de la pensée contemporaine.

${ }^{8}$ Pour un approfondissement de la pensée de Lachelier, il me semble intéressant de renvoyer le lecteur à l'ouvrage de G. Séailles intitulé La philosophie de Jules Lachelier.
} 
aspects très importants pour une philosophie de la subjectivité et ils seront explorés en profondeur par Jean Nabert. Plus loin, Lachelier continue ainsi :

Nous accomplissons, en un mot, une destinée que nous avons choisie, ou plutôt que nous ne cessons pas de choisir : pourquoi notre choix n'est-il pas meilleur, pourquoi préférons-nous librement le mal au bien, c'est ce qu'il faut selon toute apparence, renoncer à comprendre. Expliquer, d'ailleurs, serait absoudre, et la métaphysique ne doit pas expliquer ce que condamne la morale. (Jules Lachelier, 1949 : 70)

Pour Lachelier donc, une philosophie de la subjectivité ne rayonne pas à partir de l'analyse de faits extérieurs mais plutôt depuis la fondation métaphysique du moi par une méthode de réflexion à la seconde puissance, c'est-à-dire à travers un retour de la pensée sur elle-même. Son parcours spéculatif met en évidence le fait qu'une double science régit toute connaissance de ce qui compose la dynamique de notre vie intérieure. Voici comment Lachelier achève son article :

L'homme intérieur est double, et il n'y a rien d'étonnant à ce qu'il soit l'objet de deux sciences qui se complètent l'une l'autre. La psychologie a pour domaine la conscience sensible : elle ne connaît de la pensée que la lumière qu'elle répand sur la sensation ; la science de la pensée en elle-même, de la lumière dans sa source, c'est la métaphysique. (Jules Lachelier, 1949 : 71)

Après Lachelier, plusieurs philosophes de la subjectivité et de l'intériorité réflexive ont manifesté leur génie propre et leur orientation spécifique mais c'est Jean Nabert, un des derniers représentants de cette philosophie, qui fera avancer la réflexion de Lachelier vers une philosophie centrée sur le moi et la liberté avec une analyse très pointue sur la question du mal liée à une causalité impure de la conscience. 
La première tâche d'une philosophie réflexive est pour Nabert la fondation du sujet capable de se réapproprier ses actes par un mouvement réflexif de retour sur soi vers la découverte d'une causalité originaire de la conscience source de tout acte volitif.

Laissant de côté les philosophies où c'est l'absolu qui se réfléchit dans le mouvement d'une conscience particulière, Nabert se concentre sur celles où la réflexion constitue d'abord le sujet luimême et le ressaisit ensuite à l'arrière de ses actes et de ses productions pour s'en approprier la signification. Nabert admet que l'acte initial puisse être conçu de façon différente, comme acte de juger, comme intellection des signes, comme aperception immédiate du moi dans le fait primitif de l'effort. (Paul Ricœur, 1986 : 9)

À partir de situations externes à nous mais qui ont un impact sur notre vie, Nabert oriente notre pensée vers la considération de nos états intérieurs à travers une prise de conscience réflexive de soi qui est le signe d'une relation de soi à soi. Cette analyse philosophique des états d'âme, des passions, des désirs, des déceptions et des culpabilités, on la retrouve aussi en littérature et tout particulièrement dans le roman psychologique bien que celui-ci ne soit pas une particularité littéraire spécifiquement française.

L'itinéraire spéculatif de Nabert explore la liberté et le mal comme expressions du sujet dans sa modalité d'exister. Il va au-delà des échecs objectivables dans les faits historiques, pour considérer un échec plus profond et plus radical qui est constitutif du moi dans sa réalité métaphysique. Selon la conception de Nabert, le véritable drame consiste dans le fait que le moi est en quelque sorte divisé dans la malheureuse condition de l'inégalité de son être à lui-même. 
Nabert oriente sa réflexion sur des situations humaines douloureuses comme la faute, l'échec et la solitude et à partir d'elles il cherche une compréhension de la conscience et de son être véritable. Il insiste sur le fait que le sentiment de culpabilité est la prise de conscience d'une vulnérabilité ontologique d'un moi qui a échoué. Bien que la culpabilité révèle une transgression des normes morales, elle se manifeste comme expression de la douloureuse fracture intérieure d'un moi qui ne correspond pas à lui-même. La solitude, la faute et l'échec sont vus par Nabert dans son Éléments pour une éthique comme l'expression d'un « sentiment fondamental qui traduit l'inégalité de nous-mêmes à nous-mêmes ou de l'être que nous devenons à notre être véritable »(Jean Nabert, $1962: 61)$.

Dans son Essai sur le mal, Nabert analyse l'expérience humaine caractérisée par le drame intérieur engendré par l'opposition entre l'unité exigée par le moi et la rupture de division du même moi. Dans tout le parcours nabertien, le problème du mal est donc vu comme produit de la liberté en tant que causalité originaire de la conscience. La liberté en tant qu'acte qui détermine originairement le mal, révèle l'exercice d'une causalité spirituelle qui réside dans le moi. Voici que le moi, face à la déroutante réalité du mal, se caractérise comme causalité spirituelle impure.

En définissant la causalité spirituelle du moi, Nabert écrit ainsi dans son Essai sur le mal:

Qu'est-ce que donc une causalité spirituelle - à la différence d'une causalité psychique dont on est en droit de penser qu'elle est toujours présente, à quelque degré, chez les êtres vivants sinon une causalité 
dont l'acte, générateur de possibles, s'éclaire, se connaît, par les motifs qui en épanouissent la signification, permettent un jugement, et laissent quelque intervalle, si court qu'on le conçoive, entre le jaillissement de l'idée et son actualisation? (Jean Nabert, 1970 : 74 75)

Ce sont ces choix qui permettent de possibles trahisons ou négations qui ne sont pas en dehors du moi mais générées par le moi lui-même. Elles sont le produit d'une causalité impure qui trouve son origine dans le moi et qui rend le moi inégal à lui-même.

Dans cette perspective, Nabert introduit la notion d' « injustifiable » pour identifier le mal. L'injustifiable pour Nabert n'est pas quelque chose en soi d'objectivable mais plutôt un acte dans les profondeurs même de la conscience et qui agit en la niant par une sorte de trahison.

Pour comprendre l'itinéraire nabertien, il faut entreprendre avec lui un retour sur soi, méthode spécifique de la philosophie réflexive, afin de retrouver l'intimité spirituelle du moi. Bien entendu, en disant cela, je ne veux pas orienter ma réflexion vers des considérations morales ou psychologiques. Cependant, l'itinéraire méthodologique nabertien autour de questions comme la liberté et le mal, nous conduirait inévitablement vers l'élaboration d'une philosophie morale. Et puis encore, le sentiment d'échec qui surgit suite à un retour réflexif sur soi à partir des actes accomplis et considérés ensuite comme transgression d'une norme inscrite dans le moi pur, nous conduirait aussi vers une psychologie analytique ou cognitive dont le champ d'investigation serait bien différent de celui d'une philosophie de la subjectivité et de l'intériorité réflexive. 
Nabert va bien au-delà d'un psychologisme pur ou d'un moralisme philosophique, car pour lui le problème du mal comme injustifiable est plus profond que la transgression d'une norme morale. Pour lui l'injustifiable est un mal radical produit par une causalité impure dont le moi est la source.

Il n'est pas question de creuser la possibilité de connaissance des jugements moraux comme voudrait le débat entre cognitivistes et non cognitivistes dans le champ méta-éthique, mais plutôt d'examiner le moi dans la possibilité éventuelle de se comprendre.

L'orientation commune de différents penseurs qui revendiquent la méthode réflexive vise la saisie d'une conscience pure mais ce sera Nabert, qui à mon sens, atteint avec une analyse assez dense, le fondement d'une philosophie française de la subjectivité réflexive. Voici que Nabert, dans son article intitulé « La philosophie réflexive », écrit ainsi :

La réflexion ramène essentiellement au sujet, aux opérations dont il répond, dans une perspective d'immanence [...]. Le propre de la réflexion ainsi comprise, c'est de toujours considérer l'esprit dans ses actes et dans ses productions, pour s'en approprier la signification, et, d'abord, essentiellement, dans l'acte initial par lequel le sujet s'assure de soi, de son pouvoir, de sa vérité. (Jean Nabert, 1957 : 19)

Cela dit, peut-on avoir une vision naïve selon laquelle cette philosophie de la subjectivité qui adopte, comme on l'a vu, la méthode réflexive, soit restée totalement inconnue à toutes formes de production de l'esprit humain ? Mon idée est celle de mettre en lumière la présence de cette pensée philosophique en littérature et notamment dans la production littéraire de Marcel Proust. 


\section{Marcel Proust et la philosophie constitutive de son époque}

Littérature et philosophie peuvent faire chacune l'objet d'une étude analytique relative à leur statut propre, mais quand cette étude concerne la production de Marcel Proust, alors l'interaction de ces deux disciplines révèle toute sa complexité avec des nuances touchant le vaste panorama des sciences humaines et tout particulièrement la psychologie, la psychanalyse et la métaphysique.

Une lecture approfondie de l'œuvre de Proust, universellement connu par son chef d'œuvre À la recherche du temps perdu, nous permettra de repérer des éléments de philosophie réflexive harmonieusement dissimulés dans une prose profondément élaborée. Il est, en effet, intéressant de mettre en évidence l'aspect de «la conscience de soi » dans l'œuvre de Proust et sa méthode réflexive qui semble traduire en prose une philosophie du sujet sur le modèle de la méthode employée par la pensée que je suis en train d'évoquer.

L'œuvre littéraire de Proust naît et se développe dans une période où la philosophie réflexive française connaît tout son rayonnement. Notre auteur avait eu la possibilité lors de ses études universitaires, de creuser la pensée philosophique de son temps. Son cheminement littéraire est sans doute imprégné de cette méthode philosophique qui puise ses racines dans l'activité réflexive de la conscience dans sa tentative de se saisir et de mieux se connaître.

Proust est sans doute un des plus grands observateurs analytiques d'objets ou d'événements ou encore de souvenirs à travers lesquels il cherche une compréhension de l'esprit dans sa faculté de penser. Bien souvent le narrateur 
passe $\mathrm{du}$ «je » au «nous» pour prouver comment certaines expériences ou intuitions personnelles peuvent être appliquées à tout homme dans sa capacité subjective de fonctionner au niveau de la pensée. Son style littéraire très nuancé dans toutes sortes de détails, ses phrases très longues et complexes, révèlent la personnalité pas du tout superficielle d'un auteur en quête infinie de sens.

En ciblant un objet (la madeleine, la sonate de Vinteuil, les clochers de Martinville, les parvis inégaux de la cour de l'hôtel de Guermantes, etc.), le narrateur fait un retour sur soi pour se réapproprier son propre moi à travers une réflexion sur la réflexion. Voici une démarche réflexive à la seconde puissance sur le modèle de la philosophie de son temps.

Intéressant ce que le narrateur proustien écrit dans Le temps retrouvé par rapport à l'effort pour se réapproprier un instant du passé lié à une sonnette :

Pour tâcher de l'entendre de plus près, c'est en moi-même que j'étais obligé de redescendre [...] Quand elle avait tinté j'existais déjà, et depuis pour que j'entendisse encore ce tintement, il fallait qu'il n'y eût pas eu discontinuité, que je n'eusse pas un instant cessé, pris le repos de ne pas exister, de ne pas penser, de ne pas avoir conscience de moi, puisque cet instant ancien tenait encore à moi, que je pouvais encore le retrouver, retourner jusqu'à lui, rien qu'en descendant plus profondément en moi. (Marcel Proust, 1989 : 623-624)

Une recherche soignée et approfondie devrait mettre en évidence les descriptions du narrateur en lien avec toute démarche de réappropriation du moi vers une auto-conscience avertie et à travers une esthétique littéraire remarquablement complexe et originale. En effet, toute la production littéraire de Proust opère une décomposition extrêmement minutieuse de l'esprit humain qui 
mérite d'être étudiée dans sa méthode spécifique et dans son interaction avec la philosophie de la subjectivité réflexive de son temps.

Sans négliger les aspects liés à l'esthétique propre de Proust en tant qu'écrivain et à toute sa technique qui est celle d'un romancier très original, il serait intéressant de mettre en valeur la spécificité de sa méthode d'analyse inspirée du mouvement psychanalytique de son temps assez centré sur le vécu des états psychologiques et le mode de fonctionnement de l'inconscient et aussi de la philosophie dont il fut profondément imprégné.

Il y a plusieurs thèmes dans l'œuvre de Proust qui méritent une attention particulière, surtout ceux relatifs à la manière dont le narrateur prend conscience de soi par un mouvement réflexif à partir de certaines expériences vécues. Le désir, l'amour, la jalousie, l'attente, l'illusion, le regard, l'art, sont tous des sujets d'une ampleur infinie en ce qui concerne l'impact qu'ils exercent sur l'âme du narrateur.

La conscience de soi chez Proust n'est pas le résultat d'un fait immédiat mais elle implique une démarche faite d'intermittences, d'abandons et de reprises. Parfois elle semble s'éclairer d'emblée par une saisie intuitive, parfois elle semble se plier à la méthode inductive sur le modèle de Jules Lachelier et parfois elle se voit investie dans une analyse rétrospective et un effort réflexif sur le modèle de Maine de Biran ou d'autres penseurs qui adoptent une méthode similaire. La complexité de sa manière de procéder reflète les mouvements de tout esprit humain qui demeure en soi insaisissable, imprévisible et source infinie de nouveautés au-delà de tout conditionnement déterministe. En effet, 
Si la philosophie est un effort de la conscience pour s'approfondir, sa méthode ne pourra être qu'une réflexion qui, par-delà des contenus de conscience toujours mondains, s'efforcera de saisir les intentions mêmes de l'activité qui les élabore. L'esprit ne peut s'atteindre que réflexivement. [...] C'est donc en réfléchissant à partir des signes produits, que la conscience s'éprouvera et tentera de se connaître dans sa dimension spirituelle. (Gabriel Madinier, 1953 : 6)

La philosophie réflexive, dans sa méthode d'analyse à la seconde puissance, vise à identifier en nous une causalité purement spirituelle comme véritable principe de nos affirmations métaphysiques.

La célèbre mémoire involontaire mérite un approfondissement cognitif dans sa manière de se manifester chez le narrateur. La philosophie qui se dégage de son œuvre pouvait être le reflet de la lecture des auteurs assimilés pendant sa formation universitaire mais aussi la manifestation d'une pensée assez personnelle bien que dans la mouvance de la pensée de ses contemporains. La conscience de soi, la mémoire et la dimension de la temporalité chez Proust ouvrent des pistes de grand intérêt philosophique et littéraire, éclairées par la pensée d'Henri Bergson ${ }^{9}$ puis par Paul Ricœur, surtout dans Temps et récit.

L'œuvre de Proust est dominée par la distinction entre mémoire volontaire et mémoire involontaire mais dans les deux cas, l'auteur fait une démarche analytique de réflexion vers les profondeurs de la conscience à la conquête du moi pur. Cette démarche, qu'elle soit adoptée dans sa production littéraire ou qu'elle soit un itinéraire spéculatif de toute philosophie de l'esprit à la seconde puissance, demeure toujours inachevée car le moi pur, de par sa nature

\footnotetext{
${ }^{9}$ Par Matière et mémoire, Bergson a donné un caractère totalement spirituel à la mémoire liée au temps contre la res cogitans cartésienne liée à la substance. On lirait avec profit l'œuvre d'Albert Thibaudet, élève et ami de Bergson, intitulée Le bergsonisme.
} 
spirituelle, est toujours insaisissable. En effet, la mémoire involontaire chez Proust est seulement le moteur déclenchant le parcours de la mémoire volontaire. La plupart des auteurs qui ont publié des études sur Proust, s'arrêtent à l'aspect immédiat de la mémoire involontaire. Il est par contre intéressant de montrer que Proust engage une analyse réflexive non pas seulement à partir de la mémoire volontaire mais aussi à partir de la mémoire involontaire :

Mais à l'instant même où la gorgée mêlée des miettes du gâteau toucha mon palais, je tressaillis, attentif à ce qui se passait d'extraordinaire en moi. Un plaisir délicieux m'avait envahi, [...] Je sentais qu'elle était liée au goût du thé et du gâteau, mais qu'elle le dépassait infiniment, ne devait pas être de même nature. D'où venaitelle ? Que signifiait-elle ? Où l'appréhender? Je bois une seconde gorgée où je ne trouve rien de plus que dans la première, une troisième qui m'apporte un peu moins que la seconde. Il est temps que je m'arrête, la vertu du breuvage semble diminuer. Il est clair que la vérité que je cherche n'est pas en lui, mais en moi. [...] Je pose la tasse et me tourne vers mon esprit. C'est à lui de trouver la vérité. (Marcel Proust, 1989 : 44-45)

La mémoire involontaire se déclenche sans aucun apport conscient et fait resurgir de manière immédiate un souvenir oublié ou refoulé. En revanche, le narrateur, dans l'épisode de la madeleine évoqué dans le passage en haut, fait un effort pour déchiffrer les souvenirs et pour trouver en lui-même la vérité. Voici que la mémoire involontaire la plus connue de l'œuvre de Proust n'est pas tout à fait involontaire mais moteur réflexif vers une recherche des profondeurs du moi.

Nous retrouvons une méthodologie similaire dans la thèse de Madinier, Conscience et mouvement, selon laquelle l'analyse réflexive et la vie de la conscience sont régies par une intentionnalité motrice. Il s'agit de toute une 
orientation de pensée initiée par Maine de Biran et inspiratrice, avec des acceptions différentes, de plusieurs penseurs et écrivains.

La démarche de Proust est en quelque sorte assez proche de celle de Maine de Biran qui a recours à l'introspection pour la recherche de la vérité ${ }^{10}$.

Par l'effort analytique et par une réflexion à la seconde puissance, le narrateur proustien devient de plus en plus conscient des mouvements de l'âme et de son être personnel. Maine de Biran affirme que :

L'effort emporte nécessairement avec lui la perception d'un rapport entre l'être qui meut ou qui veut mouvoir, et un obstacle quelconque qui s'oppose à son mouvement; sans un sujet ou une volonté qui détermine le mouvement, sans un terme qui résiste, il n'y a point d'effort, et sans effort, point de connaissance, point de perception d'aucune espèce. Si l'individu ne voulait pas ou n'était pas déterminé à commencer de se mouvoir, il ne connaîtrait rien. Si rien ne lui résistait, il ne connaîtrait rien non plus, il ne soupçonnerait aucune existence, il n'aurait pas même d'idée de la sienne propre. (Pierre Tisserand, $1922: 26$ )

Voici que la vraie connaissance implique un mouvement vers un dépassement de tout ce qui empêche une compréhension aussi profonde que possible de la réalité, des autres, mais surtout de soi-même. Proust, en partant de situations personnelles assez variées, comme, par exemple, le désir, la jalousie, la déception ou encore la séduction de la beauté féminine en tant qu'objet éloigné et pour cela encore plus attirant, se livre à des considérations d'ordre général qui touchent le «nous », la conscience humaine, le mode de fonctionnement de notre esprit et sa

\footnotetext{
${ }^{10}$ On lirait avec profit La recherche d'une première vérité mais aussi d'autres écrits de Jules Lequier, un penseur peu connu mais très intéressant en ce qui concerne la métaphysique du moi à travers une analyse tourmentée autour de la question de la liberté.
} 
possible capacité de se comprendre. Ceci est dans la mouvance philosophique de son temps et c'est bien l'orientation philosophique de la philosophie réflexive que je suis en train de considérer. Or, dans cette mouvance, Proust cherche à saisir cette soif d'infini qui est en lui, il cherche à la comprendre jusqu'aux racines, dans les profondeurs du moi, et ceci par des réflexions minutieuses sur des faits mondains, concrets, finis mais aussi sur des réalités spirituelles qui transcendent l'objectivable et qui révèlent en même temps la nature de notre conscience humaine située entre immanence et transcendance.

Plus tard, Nabert rendra encore plus explicite la préoccupation de toute la philosophie réflexive, à savoir ce besoin de la conscience de se comprendre. Dans son manuscrit « La conscience peut-elle se comprendre ? » nous lisons ainsi :

À la vérité, tout le problème de la transcendance ou de l'immanence n'a de sens que du point de vue d'un réalisme de l'absolu ou de l'intelligible: alors on peut se complaire autant qu'on le veut, à conjuguer la transcendance et l'immanence : c'est une dialectique dont on perce vite à jour le procédé. [...] Il apparaît au contraire que l'immanence et la transcendance sont deux manières dont la conscience se faisant acte, dans son effort pour se comprendre, peut envisager son rapport avec elle-même. (Jean Nabert, 1966 : 414-415)

Bien entendu, on ne dirait jamais que Nabert ait influencé Proust, car le décalage temporel entre les deux ne le permettrait pas, mais cet auteur est d'importance non négligeable pour comprendre la pensée réflexive dont l'œuvre littéraire de Proust semble être imprégnée. Il ne s'agit pas de prouver que Proust ait voulu reproduire dans son œuvre la pensée des philosophes qu'il a connus en étudiant leurs écrits mais plutôt de montrer que l'originalité de son style et de sa méthode a fleuri dans un contexte philosophique qui donne le primat à la vie de la conscience. Dans ce contexte, la conception du temps, du souvenir, des émotions, 
du mal et de la souffrance ainsi que le rôle de la mémoire sont élaborés comme des états intérieurs et subjectifs et non seulement comme des faits concrets et objectivables, pour les voir transposés dans la production littéraire proustienne avec un génie artistique fascinant.

\section{Conclusion}

Une recherche sur la méthode réflexive dans l'œuvre de Proust mettrait en évidence non pas seulement les éléments d'analyse vers une métaphysique de la conscience présents dans sa production littéraire, mais aussi le parallélisme existant entre la philosophie réflexive française de son époque et la littérature.

Faire resurgir la philosophie réflexive qui a été pour longtemps presque oubliée, en parallèle avec l'analyse de l'œuvre de Proust, me semble une démarche assez singulière et innovatrice pour l'histoire de la littérature française contemporaine et la critique littéraire. Il est vrai qu'au-delà de toute supposition relative à une possible influence philosophique dans sa production littéraire, ce sera Proust lui-même qui nous manifestera

le sentiment d'appartenir à une génération de philosophes, qui ont été pour la plupart ses maîtres et sont encore ses contemporains : une lettre de 1904 énumère « M. Lachelier, M. Darlu, M. Boutroux » (IV, 234); une autre de 1908 juxtapose de même « un Boutroux, un Bergson » (VIII, 140). (Luc Fraisse, 2013 : 254)

Voici donc que mon étude ouvre des perspectives vers la recherche de repères d'une philosophie de la subjectivité réflexive dans l'œuvre littéraire de Proust en sachant qu'il y a énormément à creuser et que tout engagement dans ce sens restera toujours inachevé. 
Nous savons à ce jour, qu'avec la sensibilité d'un homme malade physiquement et en quelque sorte aussi frustré psychologiquement, Proust a su plonger son regard sur le monde et sur les mouvements de l'âme humaine, pour transcender la réalité matérielle vers la production artistique à travers un génie littéraire hors du commun. Ce rapport tout particulier entre notre auteur et l'œuvre d'art est le terrain sur lequel se dénoue la pensée assez complexe et difficile à systématiser d'un écrivain français de réputation mondiale. 


\section{Bibliographie}

Bergson, Henri, Matière et mémoire, Paris, Presses Universitaires de France, 2008.

Fraisse, Luc, L'éclectisme philosophique de Marcel Proust, Paris, Presses de l'université Paris-Sorbonne, 2013.

Lachelier, Jules, Psychologie et métaphysique, Paris, Presses Universitaires de France, 1949.

Lequier, Jules, Euvres complètes, Neuchâtel, Éditions de la Balconnière, 1952.

Madinier, Gabriel, Conscience et mouvement. Étude sur la philosophie française de Condillac à Bergson, Paris, Alcan, 1938.

Madinier, Gabriel, Conscience et signification. Essai sur la réflexion, Paris, Presses Universitaires de France, 1953.

Nabert, Jean, «La philosophie réflexive », dans Encyclopédie Française, t. XIX, Paris, Société nouvelle de l'Encyclopédie Française, 1957.

Nabert, Jean, Éléments pour une éthique, Paris, Aubier-Montaigne, 1962.

Nabert, Jean, Le désir de Dieu, Paris, Aubier-Montaigne, 1966.

Nabert, Jean, Essai sur le mal, Paris, Aubier-Montaigne, 1970.

Nabert, Jean, L'expérience intérieure de la liberté, Paris, Presses Universitaires de France, 1994.

Proust, Marcel, À la recherche du temps perdu, Paris, Gallimard, vol. 1, 1987 ; vol. 2, 1988 ; vol. 3, 1988 ; vol. 4, 1989.

Ricœur, Paul, « Négativité et affirmation originaire », Recherches de philosophie, 2, 1956. Republié dans Idem, Histoire et vérité, Paris, Éditions du Seuil, 1967. 
Ricour, Paul, « L'acte et le signe selon Jean Nabert », Études philosophiques, 3, PUF, Paris, 1962, pp. 339-349. Republié dans Le conflit des interprétations. Essais d'herméneutique, Paris, Seuil, 2013 [1969], pp. 289-304.

Ricœur, Paul, Temps et récit, Paris, Éditions du Seuil, vol. 1, 1983 ; vol. 2, 1984 ; vol. 3, 1988.

Ricoeur, Paul, Du texte à l'action. Essais d'herméneutique II, Paris, Éditions du Seuil, 1986.

Ricœur, Paul, Soi-même comme un autre, Paris, Éditions du Seuil, 1990.

Ricœur, Paul, «L'arbre de la philosophie réflexive », préface à J. Nabert, L'expérience intérieure de la liberté, Paris, Presses Universitaires de France, 1994.

Ricœur, Paul, Méthode réflexive appliquée au problème de Dieu chez Lachelier et Lagneau, Paris, Les Éditions du Cerf, 2017.

Robberechts, Ludovic, Essai sur la philosophie réflexive, Namur, Presses Universitaires de Namur, vol. 1, 1971 ; vol. 2, 1974.

Sartre, Jean-Paul, L'existentialisme est un humanisme, Paris, Éditions Nagel, 1946.

Séailles, Gabriel, La philosophie de Jules Lachelier, Paris, Félix Alcan, 1920.

Thibaudet, Albert, Le bergsonisme, vol. I-II, Paris, Gallimard, 1923.

Tisserand, Pierre, Euvres de Maine de Biran, tome II, Paris, Félix Alcan, 1922. 